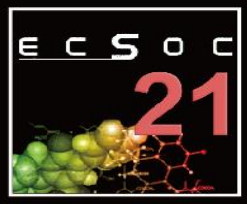

The 21st International Electronic Conference on Synthetic Organic Chemistry

\title{
Magnetic halloysite: an envirmental nanocatalyst for the synthesis of
}

\author{
benzoimidazole
}

\author{
Ali Maleki*, Zoleikha Hajizadeh \\ Catalysts and Organic Synthesis Research Laboratory, Department of Chemistry, Iran \\ University of Science and Technology, Tehran16846-13114, Iran
}

*Corresponding author. E-mail: maleki@iust.ac.ir

\begin{abstract}
In the present work, halloysite nanotubes was modified by magnetic nanoparticles and used as an efficient, eco-friendly catalyst in synthesize of benzoimidazole derivatives. This synthesized nanocomposite was characterized successfully by Fourier transforms infrared spectroscopy (FTIR), scanning electron microscopy (SEM). The catalyst can simply be separated from the reaction mixture by an external magnet and reused in several runs. Reusable and easily recoverable catalyst, use of green solvent, room temperature and mild reaction conditions are some advantages of the present work.
\end{abstract}

Keywords: Halloysite, $\mathrm{Fe}_{3} \mathrm{O}_{4}$, Nanocatalyst, Imidazole. 


\section{Introduction}

A great amount of clay minerals are existed in soils and weathered rocks that encompasses unique properties and wide applications due to their nanoscale structures [1]. Because of being biocompatible and cheap in nature, halloysite has been considered a new generation of nanostructures in material science and technology. Halloysite is ubiquitous in Australia and the United States, China, New Zealand, Mexico, and Brazil. Also, It is chemically similar to kaolinite, in which layers are separated by a monolayer of water molecules; accordingly, halloysite has a structural formula of $\mathrm{Al}_{2}(\mathrm{OH})_{4} \mathrm{Si}_{2} \mathrm{O}_{5} \cdot \mathrm{nH}_{2} \mathrm{O}$. From structurally aspect, halloysite generally appears in tubular, platy and spheroidal morphologies, but the dominant structure contains multi-layer wall of hollow tubules [2]. High surface area of halloysite is leading to be a good host for immobilizing metals to enhance their catalytic activity [3].

Nanoparticle $\mathrm{Fe}_{3} \mathrm{O}_{4}$-supported catalysts possesses unique features such as low cost, easy preparation, environmental compatibility, non-toxicity, reusability, chemical and physical stability [4].

Heterocyclic compounds, especially the nitrogen containing, are always center of attention in pharmaceutical and biological research areas. Imidazoles are one of the interesting heterocycles which employed widely in medicine chemistry and their unique biological activities such as antiinflammatory [5], anti-parasitic [6], antifungal [7], antiviral [8].

In present study we report synthesis imidazole compounds $\mathbf{3}$ via the condensation of benzaldehyde $\mathbf{1}$ and $O$-phenylenediamine $\mathbf{2}$ in mild condition and high yield by use of $\mathrm{Fe}_{3} \mathrm{O}_{4} @$ halloysite as an efficient, ecofriendly and heterogeneous catalyst (Scheme 1). 


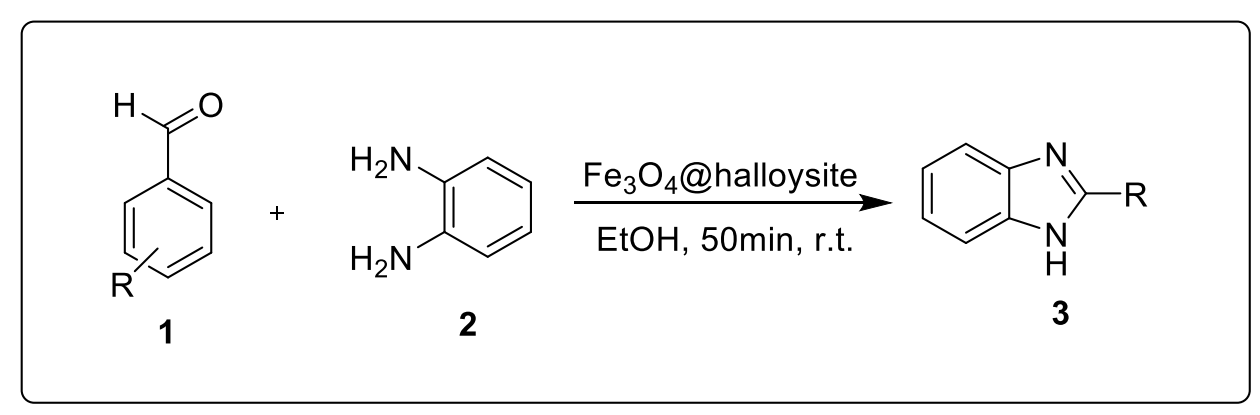

Scheme 1. $\mathrm{Fe}_{3} \mathrm{O}_{4} @$ halloysite catalyzed synthesis of imidazole compounds.

\section{Experimental section}

\subsection{General}

Reagents, solvents and chemicals were all purchased from Aldrich, Merck and Fluka. Melting points were measured with an Electrothermal 9100 apparatus and are uncorrected. Spectra of FTIR were recorded by the method of $\mathrm{KBr}$ pellet on a Shimadzu IR-470 spectrometer. SEM images were taken with Sigma-Zeiss microscope with attached camera a Numerix DXP-X10P.

\subsection{Synthesis of $\mathrm{Fe}_{3} \mathrm{O}_{4} @$ halloysite}

At first, $0.5 \mathrm{~g}$ of halloysite was added to a $200 \mathrm{~mL}$ solution of $1.165 \mathrm{~g} \mathrm{FeCl}_{3} .6 \mathrm{H}_{2} \mathrm{O}$ and $0.429 \mathrm{~g}$ of $\mathrm{FeCl}_{2} \cdot 4 \mathrm{H}_{2} \mathrm{O}$ under $\mathrm{N}_{2}$ and $60{ }^{\circ} \mathrm{C}$. To prepare iron oxide, $20 \mathrm{~mL}$ solution of $\left(\mathrm{NH}_{3}, \mathrm{H}_{2} \mathrm{O}\right) 8$ $\mathrm{mol} / \mathrm{L}$ was added dropwise to the initial mixture. The $\mathrm{pH}$ of the final mixture was controlled in the range of 9-10 and stirred for $4 \mathrm{~h}$. Then, it was washed with distilled water 2 times. Finally, the obtained nanocomposite was dried at $100{ }^{\circ} \mathrm{C}$ for $3 \mathrm{~h}$. 


\subsection{General procedure for the synthesis of imidazole derivatives $\mathbf{3 a - e}$}

Initially, $O$-phenylenediamine $(1 \mathrm{mmol}, 0.181 \mathrm{~g})$, benzaldehyde $(1 \mathrm{mmol}, 0.104 \mathrm{~g})$ and $\mathrm{Fe}_{3} \mathrm{O}_{4} @$ halloysite $(0.05 \mathrm{~g})$ were mixed in ethanol. The mixture was stirred in an air atmosphere at room temperature for $50 \mathrm{~min}$. After the completion of the reaction, as indicated by TLC, the catalyst was removed easily by external magnet. The crude product was filtered and recrystallized from ethanol to give products in good to high yields.

\section{Results and discussion}

FT-IR spectroscopy was used for the synthesis confirmation $\mathrm{Fe}_{3} \mathrm{O}_{4} @$ halloysite. The broad and intense band at $3442 \mathrm{~cm}^{-1}$ was due to stretching vibrations of hydroxyl groups from iron oxide. It is needed to mention that the band of Al-O-Si related to halloysite at $536 \mathrm{~cm}^{-1}$ is overlapping with $\mathrm{Fe}_{3} \mathrm{O}_{4}$ peak at $575 \mathrm{~cm}^{-1}$ in $\mathrm{Fe}_{3} \mathrm{O}_{4} @$ halloysite.

Also, scanning electron microscopy (SEM) image of $\mathrm{Fe}_{3} \mathrm{O}_{4} @$ halloysite as obtained, where it is obvious to observe nanoscale structure of nanocomposite.

The synthesis reaction of imidazile derivatives can be done by combination of $o$ phenylenediamine with aromatic aldehyde (Table 1). The optimized conditions for the reaction was using $0.05 \mathrm{~g}$ of $\mathrm{Fe}_{3} \mathrm{O}_{4} @$ halloysite at room temperature in $\mathrm{EtOH}$ solvent. 
Table 1. Synthesis of imidazole derivatives 3a-e in optimal conditions.

\begin{tabular}{cccc}
\hline Entry & $\mathrm{R}$ & Product & Yield $^{\mathrm{a}}(\%)$ \\
\hline 1 & $\mathrm{C}_{6} \mathrm{H}_{5}$ & $\mathbf{3 a}$ & 98 \\
2 & $3-\mathrm{NO}_{2}-\mathrm{C}_{6} \mathrm{H}_{4}$ & $\mathbf{3 b}$ & 95 \\
3 & $4-\mathrm{NO}_{2}-\mathrm{C}_{6} \mathrm{H}_{4}$ & $\mathbf{3 c}$ & 93 \\
4 & $2-\mathrm{NO}_{2}-\mathrm{C}_{6} \mathrm{H}_{4}$ & $\mathbf{3 d}$ & 82 \\
5 & $4-\mathrm{CN}_{-} \mathrm{C}_{6} \mathrm{H}_{4}$ & $\mathbf{3 e}$ & 93 \\
\hline
\end{tabular}

${ }^{a}$ Isolated yields.

\section{Conclusion}

In summary, we introduced a new magnetic nanocomposite system based on halloysite. We provide a simple, green and extremely efficient protocol for the synthesis of imidazole derivatives in presence of the mentioned environmentally friendly and reusable magnetic nanocatalyst.

\section{Acknowledgements}

The authors gratefully acknowledge the partial support from the Research Council of the Iran University of Science and Technology. 


\section{References}

1. Lvov, Y., Wang, W., Zhang, L., Fakhrullin, R. Adv. Mater. 2016, $28,1227$.

2. Zhang, Y., Yang, H. Appl. Clay. Sci. 2012, 56, 97.

3. Chen, L., Zhou, C.H., Fiore, S., Tong, D.S., Zhang, H., Li, C.S., Ji, S.F., Yu, W.H. Appl. Clay. Sci. 2016, 127, 143.

4. Maleki, A., Alrezvani, Z., Maleki. S. Catal. Commun. 2015, 69, 29.

5. Trujillo, J.I., Kiefer, J.R., Huang, W., Thorarensen, A., Xing, L., Caspers, N.L., Day, J.E., Mathis, K.J., Kretzmer, K.K., Reitz, B.A. Bioorg. Med. Chem. Lett. 2009, 19, 908.

6. Quattara, L., Debaert, M., Cavier, R. Il Farmaco.1987, 42, 449.

7. Hadizadeh, F., Hosseinzadeh, H., Motamed-Shariaty, V.-S. F. Hadizadeh, H. Iran. J. Pharm. Res. 2010, 7, 29.

8. Cheng, J., Xie, J., Luo, X. Bioorg. Med. Chem. Lett. 2005, 15, 267. 